\title{
Radiological evaluation of tube depth and complications of prehospital endotracheal intubation in pediatric trauma : a descriptive study
}

\section{Simons, $\mathrm{T}$.}

\section{7-12}

Simons , T , Soderlund , T \& Handolin , L 2017 , ' Radiological evaluation of tube depth and complications of prehospital endotracheal intubation in pediatric trauma : a descriptive study

' , European Journal of Trauma and Emergency Surgery , vol. 43 , no. 6 , pp. 797-804 . https://doi.org/10.1007/s0006

http://hdl.handle.net/10138/283167

https://doi.org/10.1007/s00068-016-0758-2

publishedVersion

Downloaded from Helda, University of Helsinki institutional repository.

This is an electronic reprint of the original article.

This reprint may differ from the original in pagination and typographic detail.

Please cite the original version. 


\title{
Radiological evaluation of tube depth and complications of prehospital endotracheal intubation in pediatric trauma: a descriptive study
}

\author{
T. Simons ${ }^{1} \cdot$ T. Söderlund ${ }^{1}$ L. Handolin ${ }^{1}$
}

Received: 11 September 2016 / Accepted: 29 December 2016 / Published online: 27 January 2017

(C) Springer-Verlag Berlin Heidelberg 2017

\begin{abstract}
Purpose Pediatric prehospital endotracheal intubation (PHETI) is a difficult and rarely performed procedure that remains the gold standard for prehospital airway management when ventilation and/or anesthesia is required, but high complications rates, including malposition continue to concern. We reviewed the experience in our institution of pediatric intubations with particular emphasis on the position of the endotracheal tube (ETT) tip within the trachea and related complications.

Method Intubated pediatric patients presenting directly from the scene to our level 1 trauma center, between 2006 and 2014, were included in our study. Patient records and radiographs were retrospectively reviewed to identify the ETT tip-to-carina distance and possible intubation-related complications. ETT tips identified beyond the carina on radiographs or by clinical diagnosis were defined as misplaced. Because head movement causes a significant ETT movement within the trachea, which is age related, we also defined ETT tip placement (1) less than $2 \mathrm{~cm}$ above the carina in children younger than 8 and (2) less than $3 \mathrm{~cm}$ above the carina in children 8 years or older as "near miss" intubations.

Results From a total of 34 cases, ETT misplacement was identified in seven cases. Diagnosis was made radiologically in five cases and clinically in two cases. Four of these patients had left lung atelectasis due to tube misplacement. Tube thoracotomy was performed in two of these patients
\end{abstract}

T. Simons

tomi.simons@hus.fi

1 Department of Orthopedics and Traumatology, Helsinki University Central Hospital and University of Helsinki, Topeliuksenkatu 5, 00260 Helsinki, Finland without concurrent evidence of chest injury. "Near miss" intubations accounted for $7 / 9$ and $9 / 25$ in children $<8$ years and $\geq 8$ years old, respectively, totaling $16 / 34$, with two of these leading to late displacements.

Conclusions Pediatric endotracheal tube intubation carries a high rate of tube malposition and left lung atelectasis in our experience of pediatric trauma patients, with less than a third of ETTs placed in a safe position.

Keywords Pediatric intubation - Pediatric prehospital endotracheal intubation $\cdot$ Safe zone $\cdot$ Tip-to-carina distance $\cdot$ Near miss intubation $\cdot$ Complication

\section{Introduction}

Respiratory arrest is a primary cause of mortality in critically ill and injured pediatric patients [1]. Securing safe and effective airway control is a priority in the prehospital setting. Prehospital endotracheal intubation (PHETI) is considered by many the gold standard of airway management. It provides the most secure and effective airway in pediatric patients according to the European Resuscitation Guidelines [2]. This assertion remains, however, at the center of debate and controversy as research has failed to show benefit from endotracheal intubation (ETI) when compared to bag-valve-mask ventilation (BVM) in the prehospital setting, including in head injured patients $[3,4]$. A higher mortality rate has also been related to PHETI patients compared to hospital intubations or no intubation [5]. Despite these findings, endotracheal tube (ETT) intubation is still the practiced norm in many emergency services [1]. Therefore, research into the optimal form of pediatric prehospital airway management still remains a top research priority [6]. 
Injured children present a particular challenge to the emergency medical services as pediatric intubations are rare high-skill procedures performed under suboptimal and emotive circumstances. Anatomic differences between adults and children, unfamiliar drug dosages, as well as inappropriately sized equipment, have led to concern over the safety and necessity of performing this potentially hazardous high-risk emergency procedure. Research into the optimal form of pediatric prehospital airway management has led to several studies looking into this controversy [7].

Children have short tracheas and, therefore, are at greater risk of misplacement of the ETT during intubation. The importance of intubation depth in children is often emphasized, but no gold standard could be identified in the literature. In adults, the Goodman's criteria recommend ETT tip placement $5 \mathrm{~cm} \pm 2 \mathrm{~cm}$ above the carina [8]. For pediatric patients, recommendations for tube tip placement $2 \mathrm{~cm}$ above the carina have been made and this recommendation has been supported by recent studies looking at pediatric ETT insertion depth [9-11].

Clinical experience from our institution suggests that ETTs are commonly placed too distally in the short tracheas of pediatric patients. The aim of this study was to identify the position of the tip of the ETT within the trachea of pediatric trauma patients presenting to our tertiary trauma center and identify associated complications and potential pitfalls of pediatric prehospital endotracheal intubations (PHETIs).

\section{Materials and methods}

Study type: retrospective review of medical records.

\section{Setting}

The Helsinki University Hospital's trauma unit is a tertiary referral trauma center with a population catchment area of about 2 million in southern Finland. The unit primarily treats adult blunt trauma patients, but severely injured children or children with suspected head injury also present to our institution as per local emergency medical response protocols. The regional emergency medical service consists of an anesthetist-staffed ambulance and an anesthetiststaffed helicopter in addition to EMT-(emergency medical technician) staffed ambulance service. Physician assistance is readily available for intubating patients in the urban surroundings close to Helsinki, but in rural areas, intubations may have to be performed by EMT personnel.

Since January 2006, all trauma admissions to the Helsinki University Hospital's trauma unit with a new injury severity score (NISS) of greater than 15 have been entered into the Trauma Registry of the Helsinki University
Hospital trauma center. This prospectively collected registry was used to retrospectively identify all intubated patients under the age of 16 years that presented directly from the scene to our trauma center between January 2006 and December 2014. Patients arriving as hospital transfers were excluded from the study even if intubated at the scene, as manipulation of the ETT at the referring hospital could not be ruled out. Patients without radiological confirmation of the position of the ETT on admission were also excluded. Ethical approval for the study was granted by the Helsinki University Central Hospital review board.

\section{Data collection}

Patient notes and radiological investigations were reviewed and relevant data were collected. This data included epidemiological data, such as age, sex, height, and weight, as well as injury-related parameters, such as mechanism of injury, ISS (Injury Severity Score), NISS (New Injury Severity Score), and initial on-scene GCS (Glasgow Coma Scale) [12-14]. Factors related to the intubation, such as indication, by whom, tube insertion depth and size were retrieved from the patient records. Patient vitals and gas exchange parameters, including arterial blood gas analysis results as well as time delays, were recorded. Patient survival and length of hospital stay were also noted. Intubation tube depth was compared to the pediatric advanced life support (PALS) manual's recommended lipline ranges for children under 2 years and using the [age (years) $/ 2+12$ ] formula for older children $[2,15]$.

Radiographs from the index admission were reviewed and the endotracheal tube tip-to-carina distance was measured from admission plain chest radiographs and/or CT scans using the AGFA IMPAX 6 PACS (picture archiving and communication system) software. We defined any ETT tip placement beyond the bifurcation of the carina as misplaced. This could be based on radiographic confirmation or clinical diagnosis based on a lack of or decreased breath sounds on chest auscultation leading to ETT pull back with subsequent radiography.

The previous work on the safe placement of ETTs in children has looked at the "tube tip-to-carina" distance and has shown marked movement in the tip of the ETT with head movement in extension and flexion [16-18]. The magnitude of this movement varies with the child's age, but an approximate $2 \mathrm{~cm}$ of movement can be shown in all children and this increases to about $3 \mathrm{~cm}$ by age $8[19,20]$. To prevent inadvertent endobronchial intubation or displacement during patient handling, this movement should be accounted for in the ETT tip-to-carina safety margin. We therefore defined any (1) ETT tip placement less than $2 \mathrm{~cm}$ above the carina in children younger than 8 years as well as 
(2) any ETT tip less than $3 \mathrm{~cm}$ above the carina in children 8 years or older as "near miss" intubations.

No specific proximal safety margin for the ETT position was defined as the vocal chords are not visualized on plain radiography. Position of the ETT on admission radiography and radiographic evidence of intubation-related complications such as atelectasis, pneumothorax, and aspiration were recorded. Placement of tube thoracotomies was also recorded as well as delayed ETT displacements and manipulations following admission.

Due to the nature of the study and the small patient numbers, we only used descriptive statistics to express our results.

\section{Results}

\section{Basic epidemiology}

For the 8-year study period, a total of 34 intubated pediatric patients were identified from the trauma register with an average age of 10 years (median of 12 years). Male patients accounted for $23(68 \%)$ cases. Five patients died within 30 days of injury at an average of $13 \mathrm{~h}$ from admission. The average length of stay (LOS) was 6 days in the intensive care unit (ICU) and 17 days in total. The patients had a mean ISS of 30 and NISS of 37 and initial GCS of 6. Mechanism of injury is shown in Table 1.

The most common indication for intubation was airway protection due to reduced GCS in $88 \%$ of patients, while other indications included: facial injury or vomiting (3 patients) and pain control (1 patient). Intubations were performed by the acute care anesthetist of the helicopter service (24 patients) or the acute care anesthetist of the ambulance service (10). Three patients were younger than 2 years of age and the only non-cuffed ETT was used in one of these cases. For $91 \%$ of cases, the ETTs were placed more than $1 \mathrm{~cm}$ deeper than the PALS recommended lipline (average $2.9 \mathrm{~cm}$ deeper; range 1-7 cm), while in the remaining 9\%, the ETT was within $1 \mathrm{~cm}$ of the recommendation.

Table 1 Mechanism of injury

\begin{tabular}{lc}
\hline Mechanism of injury & $n=34$ \\
\hline Motor vehicle accident & 15 \\
Fall from height & 5 \\
Sport & 5 \\
Bicycle & 4 \\
Pedestrian & 4 \\
Other (battered child) & 1 \\
\hline
\end{tabular}

The mechanism of injury of the included 34 pediatric patients

\section{Radiology and endotracheal tube placement}

The primary radiological investigation was performed at an average of $37 \mathrm{~min}$ from arrival to the trauma unit. This primary investigation was either a chest radiograph (16 patients) or a trauma CT scan (18 patients). Eleven patients went on to have a secondary investigation in the emergency department following their primary investigation. A total of 26 patients had CT scans done. Due to the greater accuracy of CT scans, measurements of the ETT tip-to-carina distance from CT scans were used in preference to chest radiographs (if both available), as long as no ETT manipulation had taken place between the investigations.

\section{Misplaced}

A total of $7 / 34(21 \%)$ patients had tube misplacement beyond the carina as determined by clinical or radiological examination and 4/34 (12\%) patients had left lung atelectasis as a complication of endobronchial intubation. Misplacements occurred in $2 / 9$ (22\%) patients younger than 8 years and in 5/25 (20\%) patients 8 years or older.

Radiological examination identified 5/34 (15\%) patients with ETT tip distal to the carina. Three of these had cuffs obstructing the contralateral main bronchus with two of these patients having contralateral lung atelectasis on radiological examination (see Fig. 1). In addition, one patient had left lung atelectasis despite right endobronchial intubation without cuff obstruction of the left main bronchus. Radiographic diagnosis of misplacement was made on chest X-ray and CT in two and three cases, respectively. All ETT misplacements identified entered the right main bronchus. Concern regarding ETT placement was raised in one case by the performing physician, but nevertheless the ETT remained misplaced on admission.

Clinical diagnosis of ETT misplacement through lack of breath sounds on auscultation was made in 2/34 (6\%) patients and their ETTs were pulled back prior to radiological examination. One of these patients had complete left lung atelectasis that resolved quickly on a ventilator and was therefore presumed to have been caused by cuff blockage of the left main bronchus. The other patient's ETT was pulled back more than $4 \mathrm{~cm}$ (originally being $7 \mathrm{~cm}$ deep to PALS lipline recommendation), but radiography did not reveal any associated complication.

\section{Near miss}

Based on our assigned "near miss" criteria in patients younger than 8 years, 7/9 patients (78\%) had "near miss" intubations. For patients aged 8 years or older, an ETT tipto-carina distance of less than $3 \mathrm{~cm}$ was identified in $9 / 25$ $(36 \%)$ patients, making these also "near miss" intubations. 


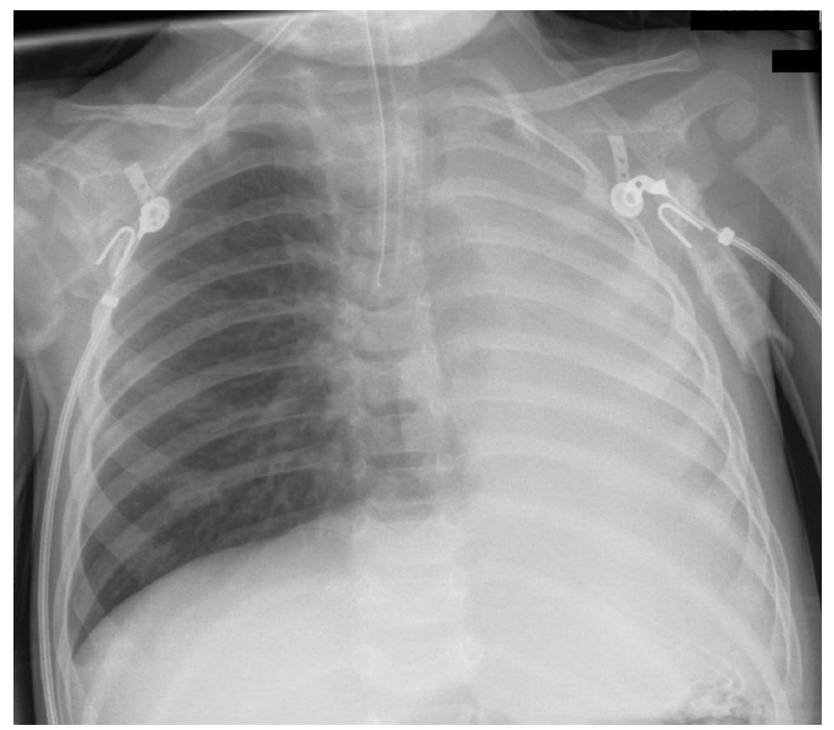

Fig. 1 Admission chest X-ray taken in the resuscitation bay of a 13-month-old boy following a fall from a height, showing left lung atelectasis associated with right endobronchial intubation

In addition, two patients with "near miss" intubations had delayed ETT displacement beyond the carina during the subsequent hospitalization. In total, 10/34 (29\%) patients had their ETT pulled back and one ETT (a "near miss" intubation) was exchanged due to air leak.

\section{Other findings}

There were 11 patients (all older than 8 years) that did not fall into our misplaced or "near miss" criteria and these were considered safe zone intubations (see Table 2). No proximal or esophageal ETT misplacements were identified. There was also no subsequent in-hospital displacements of the ETT to above the vocal chords or accidental ETT pullouts identified. Despite three patients intubated on scene due to vomiting or facial injury none of these patients had evidence of aspiration. In total, seven patients had tube thoracotomies placed, of which five patients had significant concurrent chest injuries identified. The remaining two patients with tube thoracotomies performed had radiologically identified misplaced ETTs and concurrent left lung atelectasis despite no other evidence of significant chest injury, raising suspicion that these were unnecessary performed due to ETT misplacement (see Fig. 2).

Comparison of the misplaced, "near miss" and safe zone intubations is shown in Table 3. No clear differences in the three groups can be identified, including in the arterial blood gas analysis of patients with lung atelectasis compared to those without. Fatalities occurred in each group with $2 / 7(29 \%)$ in the misplaced group, $2 / 16(13 \%)$ in the "near miss" group and 1/11 (9\%) in the safe intubation
Table 2 Comparison of findings by age

\begin{tabular}{|c|c|c|c|}
\hline & $\begin{array}{l}<8 \text { years old } \\
n=9\end{array}$ & $\begin{array}{l}\geq 8 \text { years old } \\
n=25\end{array}$ & $\begin{array}{l}\text { Total } \\
n=34\end{array}$ \\
\hline \multicolumn{4}{|l|}{ Endotracheal tube placement } \\
\hline Misplaced & 2 & 5 (2 clinical $)$ & 7 \\
\hline Lung atelectasis & 1 & 3 (1 clinical) & 4 \\
\hline Near miss & 7 & 9 & 16 \\
\hline Safe zone & 0 & 11 & 11 \\
\hline \multicolumn{4}{|l|}{ Intubation by } \\
\hline Ambulance & 5 & 5 & 10 \\
\hline $\begin{array}{l}\text { HEMS (helicopter emer- } \\
\text { gency medical service) }\end{array}$ & 4 & 20 & 24 \\
\hline $\begin{array}{l}\text { Tube depth compared to } \\
\text { PALS }\end{array}$ & $+2.0 \mathrm{~cm}$ & $+2.9 \mathrm{~cm}$ & $+2.6 \mathrm{~cm}$ \\
\hline \multicolumn{4}{|l|}{ Other variables } \\
\hline Mortality (survival time) & $2(14 \mathrm{~h})$ & $3(12 \mathrm{~h})$ & $5(13 \mathrm{~h})$ \\
\hline \multicolumn{4}{|l|}{ Length of stay } \\
\hline ICU & 3 days & 7 days & 6 days \\
\hline Hospital & 14 days & 18 days & 17 days \\
\hline
\end{tabular}

Patients are grouped by age into those younger than 8 years and those 8 years or older. Placement of the endotracheal tube (ETT) as determined on admission to the trauma unit is categorised as per our definitions for misplaced, near miss, and safe intubations. Patients with ETT misplacement diagnosed on clinical grounds are shown in brackets. Numbers given are patient numbers except those for tube depth, length of stay, and survival time which are averages. Tube depth is given as the observed ETT lipline compared to the paediatric advanced life support (PALS) recommended lipline. A positive number means that the tube is deeper than recommended. Mortality is given as the number of patients who died in each group and the survival time is an average in hours from admission. Length of stay is given in days in the intensive care unit (ICU) and hospital

group, mostly due to massive internal injuries and non-survivable head injuries. No fatality was deemed to be related to ETT misplacement. Misplaced intubations were performed by the acute care anesthetist of the ambulance service and helicopter service in 1/10 (10\%) and 6/24 (25\%), respectively. These differences are small and considering the small sample size, particularly as no injury adjusted analysis was performed, no conclusions can be drawn from these findings.

\section{Discussion}

Our results clearly highlight the difficulty of pediatric PHETI, with only $9 \%$ of pediatric patients having ETTs placed within $1 \mathrm{~cm}$ of the PALS lipline recommendation. Poor adherence to guidelines could explain the alarmingly low rate of correct ETT lipline placement seen in our case series. This finding is worrying, but consistent with the previous reports on pediatric PHETI. A recent Danish study from a Helicopter Emergency Medical Service (HEMS) 

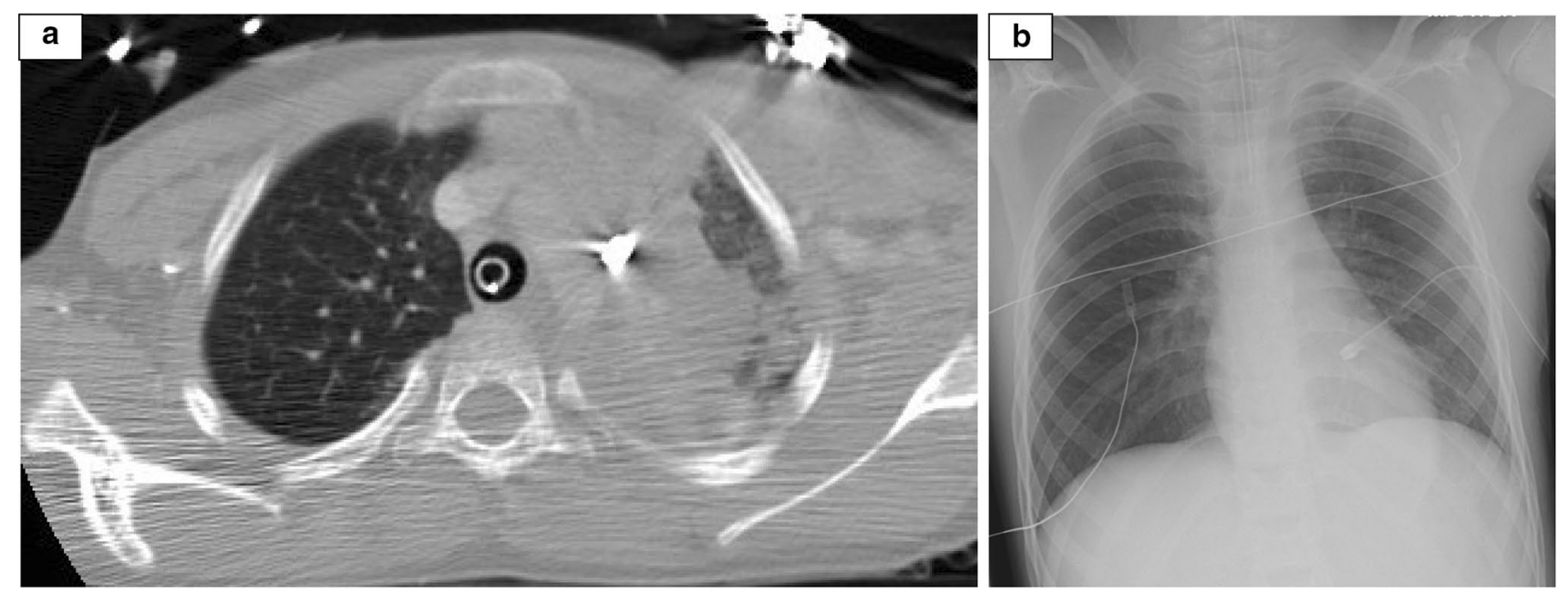

Fig. 2 Radiographs of an 11-year-old boy involved in a motor vehicle accident revealed right endobronchial intubation. Admission CT scan a shows the intubation tube in the trachea and left lung atelectasis. Note that a chest drain has been placed in the left pleural cav- ity prior to imaging due to reduced breath sounds on that side. No significant chest injury was identified and the chest X-ray b quickly resolved following endotracheal tube pull back, raising suspicion that tube thoracotomy with its added morbidity was unnecessary
Table 3 Comparison of groups by endotracheal tube position on admission

\begin{tabular}{|c|c|c|c|c|c|}
\hline Patient variables & $\begin{array}{l}\text { Misplaced } \\
n=7 \\
21 \%\end{array}$ & $\begin{array}{l}\text { Near miss } \\
n=16 \\
47 \%\end{array}$ & $\begin{array}{l}\text { Near miss } \\
<8 \text { years } \\
n=7\end{array}$ & $\begin{array}{l}\text { By age } \\
>8 \text { years } \\
n=9\end{array}$ & $\begin{array}{l}\text { Safe zone } \\
n=11 \\
32 \%\end{array}$ \\
\hline Males & $n=6$ & $n=10$ & $n=5$ & $n=5$ & $n=7$ \\
\hline Age & 9 years & 9 years & 4 years & 13 years & 13 years \\
\hline ISS & 35 & 26 & 29 & 23 & 34 \\
\hline NISS & 42 & 34 & 32 & 35 & 37 \\
\hline Initial GCS & 6 & 6 & 6 & 5 & 6 \\
\hline \multicolumn{6}{|l|}{ Intubation by } \\
\hline Ambulance & $n=1$ & $n=6$ & $n=4$ & $n=2$ & $n=3$ \\
\hline HEMS & $n=6$ & $n=10$ & $n=3$ & $n=7$ & $n=8$ \\
\hline Tube depth compared to PALS & $+3.6 \mathrm{~cm}$ & $+2.1 \mathrm{~cm}$ & $+1.7 \mathrm{~cm}$ & $+2.4 \mathrm{~cm}$ & $+2.8 \mathrm{~cm}$ \\
\hline $\mathrm{ETCO}_{2}(\%)$ & 4.5 & 4.4 & 4.6 & 4.2 & 4.6 \\
\hline $\mathrm{PaCO}_{2}(\mathrm{kPa})$ & 5.7 & 5.3 & 4.8 & 5.7 & 5.3 \\
\hline Base excess (mmol/l) & -6.0 & -3.3 & -1.8 & -4.4 & -3.3 \\
\hline \multicolumn{6}{|l|}{ Mortality } \\
\hline$n=($ survival time $)$ & $n=2(6.5 \mathrm{~h})$ & $n=2(24 \mathrm{~h})$ & $n=1(24 \mathrm{~h})$ & $n=1(24 \mathrm{~h})$ & $n=1(3 \mathrm{~h})$ \\
\hline \multicolumn{6}{|l|}{ Length of stay } \\
\hline ICU & 6 days & 5 days & 4 days & 5 days & 8 days \\
\hline Hospital & 19 days & 14 days & 16 days & 11 days & 20 days \\
\hline
\end{tabular}

Patients are grouped by endotracheal tube position as determined on admission to the trauma unit and categorised as per our definitions for misplaced, near miss and safe intubations. Near miss intubations have been further subcategorized by age into those younger than 8 years and those 8 years or older. All numbers given are averages except those denoted by " $n=$ " which are the number of patients from the group. Age is given in years. Tube depth is given as the observed ETT lipline compared to the paediatric advanced life support (PALS) recommended lipline. A positive number means that the tube is deeper than recommended. Mortality is given as the number of patients who died in each group and the survival time is an average in hours from admission. Length of stay is given in days in the intensive care unit (ICU) and hospital

ISS Injury Severity Score, NISS New Injury Severity Score, GCS Glasgow Coma Scale (given as the initial GCS on scene), HEMS Helicopter Emergency Medical Service 
setting reported that pediatric ETTs were placed too deeply in $55 \%$ of patients and that in cases with inappropriate liplines, the ETTs were placed too deeply in 96.5\% [15]. In a recent Swiss HEMS study, tube depth was also found to be deeper than recommended by the Weiss formula in $38.9 \%$ of patients despite a $15 \%$ tolerance [21].

All of our patients presented from the field and were intubated by anesthetists of the ambulance or helicopter service. Despite this, our study clearly shows that pediatric intubation carries an alarmingly high rate of misplacements and "near misses" at 21 and 47\%, respectively. A German study reporting on anesthesia trained emergency physicians showed a $12.2 \%$ complication rate, including two unrecognized endobronchial intubations in 82 patients [7]. This study estimated that each emergency physician performed only one pediatric and one infant PHETI every 3 and 13 years, respectively, raising the issue of deskilling even among physician-led emergency medical systems. Rarity of pediatric intubations has also been highlighted in the previous reports from American EMT-led emergency services. These have described malposition rates of $13 \%$ and high overall complication rates of $23-36 \%$ [22, 23].

In our study, 16/34 (47\%) ETTs met our "near miss" definition and were thus at increased risk of placementrelated complications. Adding the seven misplaced ETTs to this figure gives a total of 23/34 (68\%) poorly placed ETTs. For patients younger than 8 years, all $9(100 \%)$ had either misplaced or "near miss" intubations, highlighting the difficulty of safe PHETI in this young age group with shorter tracheas. By comparison, misplaced and "near miss" intubations accounted for 14/25 (56\%) in the 8 years or older group. Our findings emphasize the significance of younger age as a risk factor for poorly placed ETTs and associated potential complications.

Studies on the complications of pediatric PHETI typically report on the intubation success rate, number of attempts, intubation-related hypotension, desaturation, and aspiration, while malposition is reported as esophageal, hypopharyngeal, and endobronchial [24]. Our study focused on the position of the endotracheal tube within the trachea as a potential source of complications. Head movement with minimal additional head flexion could have resulted in even more misplaced/displaced intubations with five ETTs lying within $1 \mathrm{~cm}$ proximal to the carina. Despite careful patient handling, these ETTs could easily displace into the right main bronchus, as happened later to two patients in our series, increasing the total number of complicated intubations. This highlights the need to emphasize the concept of a safe tip-to-carina distance of $2 \mathrm{~cm}$ for pediatric patients younger than 8 years. The proposed safe distance of $3 \mathrm{~cm}$ proximal to the carina for older children is in accordance with the Goodman's criteria of $5 \mathrm{~cm} \pm 2 \mathrm{~cm}$ for adults. In our series, we did not identify any proximal misplacements or esophageal intubations. However, aiming to place the ETT at a more proximal location in the trachea according to our recommendation and that of PALS may increase the risk of proximal misplacement.

In our study, 4/34 (12\%) patients had clear radiographic evidence of ETT-related complications in the form of left lung atelectasis due to right endobronchial intubation. One other patient had right endobronchial intubation with left bronchial obstruction without concurrent evidence of hypoventilation of the left lung. The ETT entered the right main bronchus in all five radiographically proven endobronchial intubations identified in our study. This is explained by the anatomically more vertical decent of the right main bronchus from the carina. In addition, the tip of the ETT is cut at a 45-degree angle opening to the left, leaving the leading edge on the right to engage with the carina and direct it into the right main bronchus. Another report on the complications related to pediatric PHETI identified a rate of $19 \%$ for endobronchial intubations, $2 \%$ for esophageal intubations, $6 \%$ for tube dislodgement and one tracheal injury [25].

The four cases of endobronchial intubation with associated left lung atelectasis found in our study represent a very significant complication that is detrimental to any severely traumatized pediatric patient. A study carried out in the optimized setting of a children's hospital's emergency department resuscitation room showed a 30\% right endobronchial intubation and $4 \%$ left lung atelectasis rate [26]. These figures are comparable to our findings of $21 \%$ and $12 \%$, respectively, and represent the clinical experience of emergency pediatric endotracheal intubation from a hospital perspective. It must also be noted that in the trauma patient setting, endobronchial intubation with loss of left sided breath sounds can easily lead to unnecessary tube thoracotomy and its added morbidity as evidenced by two patients (6\%) in our series. We wish to raise awareness of left lung atelectasis as a complication in the current debate on prehospital pediatric airway management.

In addition to the importance placed on the depth of ETTs, there also remains controversy regarding the relative benefits and disadvantages of using cuffed and uncuffed ETTs in children [27]. All three patients with left lung atelectasis had cuffed tubes and it is possible that this detrimental complication could have been avoided had an uncuffed tube been used. Classically, uncuffed tubes have been preferred in children younger than 8 years as these have been shown to have a greater safety margin regarding insertion depth when compared to cuffed ETTs [27, 28].

Our patient series represents severely traumatized patients with average ISS of 30 and thus, as per local protocol, all had C-spine immobilization with a hard collar following intubation. This should prevent any significant head movement and prevent in-transit ETT displacement 
within the trachea during transport. Neck immobilization may, however, contribute to the caudal displacement of ETTs as intubation is carried out with the head in relative extension and then brought to a more neutral or even flexed position by a hard collar and spinal board. This may explain some of the increased rate of tube misplacement seen in our series of trauma patients compared to other studies looking at pediatric PHETI performed for all causes. Data on ETT misplacement rates in trauma vs non-trauma pediatric PHETI patients are limited. Comparison of EMT-performed intubations shows endobronchial intubations occurring in 7/59 (12\%) pediatric trauma PHETIs vs $18 \%$ endobronchial intubation rate in 186 mainly non-trauma pediatric PHETI $[3,23]$.

This retrospective study reports on our experience from a tertiary trauma center that receives a small number of severely injured pediatric trauma patients. Being a retrospective study relying on the previous documentation, there were deficits in the data set particularly regarding patient length. This lack of knowledge or even estimate of patient length highlights the challenges of prehospital care in selecting appropriate intubation tube sizes and depths. In addition, radiological confirmation of the position of the ETT tip only represents its position at one specific point in time. Patient handling following imaging may result in additional tube displacements particularly for "near miss" intubations. The small sample size of our study limits the generalizability of our findings, but does represent the experience of our trauma center.

\section{Conclusions}

Pediatric prehospital endotracheal tube intubation remains a challenging procedure, carrying a high rate of tube malposition and left lung atelectasis, with less than a third of ETTs placed in a safe position. We wish to draw attention to the concept of a tube tip-to-carina safety margin. We suggest that this should be $2 \mathrm{~cm}$ for children younger than 8 years and $3 \mathrm{~cm}$ for older children, to minimize "near misses" in an attempt to prevent distal misplacement and subsequent displacement of the ETT and their associated complications.

\section{Compliance with ethical standards}

Ethical approval for the current study was obtained from the Helsinki University Central Hospital review board.

Conflict of interest Tomi Simons, Tim Söderlund and Lauri Handolin declare that they have no conflict of interest.

\section{References}

1. Anders J, Brown K, Simpson J, et al. Evidence and controversies in pediatric prehospital airway management. Clin Pediatr Emerg Med. 2014;15(1):28-37.

2. Maconochie IK, Bingham R, Eich C, et al. European Resuscitation Council Guidelines For Resuscitation 2015. Resuscitation. 2015;95:223-48.

3. Gausche M, Lewis RJ, Stratton SJ, et al. Effect of out-of-hospital pediatric endotracheal intubation on survival and neurological outcome: a controlled clinical trial. JAMA. 2000;283(6):783-90.

4. Cooper A, DiScala C, Foltin G, et al. Prehospital endotracheal intubation for severe head injury in children: a reappraisal. Semin Pediatr Surg. 2001;10(1):3-6.

5. DiRusso SM, Sullivan T, Risucci D, et al. Intubation of pediatric trauma patients in the field: predictor of negative outcome despite risk stratification. J Trauma. 2005;59(1):84-90.

6. Browne LR, Shah MI, Studnek JR, et al. Pediatric research priorities in prehospital care. Prehosp Emerg Care. 2016; 25:1-6.

7. Eich C, Roessler M, Nemeth M, et al. Characteristics and outcome of prehospital paediatric tracheal intubation attended by anaesthesia-trained emergency physicians. Resuscitation. 2009;80(12):1371-7.

8. Ong KC, A'Court GD, Eng P, et al. Ideal endotracheal tube placement by referencing measurements on the tube. Ann Acad Med Singapore. 1996;25(4):550-2.

9. Bloch EC, Ossey K, Ginsberg B. Tracheal intubationin children: a new method for assuring correct depthof tube placement. Anaesth Analg. 1988;67:590-2.

10. Kim KO, Um WS, Kim CS. Comparative evaluation of methods for ensuring the correct position of the tracheal tube in children undergoing open heart surgery. Anaesthesia. 2003;58:889-93.

11. Kemper M, Dullenkopf A, Schmidt AR, et al. Nasotracheal intubation depth in paediatric patients. $\mathrm{Br} \mathrm{J}$ Anaesth. 2014;113:840-6.

12. Baker SP, O'Neill B, Haddon W, et al. The injury severity score: a method for describing patients with multiple injuries and evaluating emergency care. J Trauma. 1974;14:187-96.

13. Osler T, Baker SP, Long WB. A modification of the injury severity score that both improves accuracy and simplifies scoring. J Trauma. 1997;43:922-5.

14. Teasdale G, Jennet B. Assessment of coma and impaired consciousness. A practical scale. The Lancet. 1974;304:81-4.

15. Orf J, Thomas SH, Ahmed W, et al. Appropriateness of endotracheal tube size and insertion depth in children undergoing air medical transport. Pediatr Emerg Care. 2000;16(5):321-7.

16. Weiss M, Dullenkopf A, Böttcher S, et al. Clinical evaluation of cuff anf tube tip position in newly designed paepiatric preformed oral cuffed tracheal tube. Br J Anaesth. 2006;97:695-700.

17. Jordi Ritz EM, Von Ungern-Sternberg BS, Keller K, et al. The impact of head position on the cuff and tube tip position of preformed oral tracheal tubes in young children. Anaesthesia. 2008;63:604-9.

18. Jin-Hee K, Ro Y-J, Seong-Won M, et al. Elongation of the trachea during neck extension in children: implications of the safety of endotracheal tubes. Anesth Analg. 2005;101:974-7.

19. Weiss M, Knirsch W, Kretschmar O, et al. Tracheal tube-tip displacement in children during head-neck movement- a radiological assessment. Br J Anaesth. 2006;96:486-91.

20. Todres ID, deBros F, Kramer S, et al. Endotracheal tube displacement in the newborn infant. J Paediatr. 1976;89:126-7.

21. Schmidt AR, Ulrich L, Seifert B, et al. Ease and difficulty of prehospital airway management in 425 paediatric patients treated by a helicopter emergency medical service: a retrospective analysis. Scand J Trauma Resusc Emerg Med. 2016;24(1):22. 
22. Denver Metro Airway Study Group. A prospective multicenter evaluation of prehospital airway management performance in a large metropolitan region. Prehosp Emerg Care. 2009;13(3):304-10.

23. Ehrlich PF, Seidman PS, Atallah O, et al. Endotracheal intubations in rural pediatric trauma patients. J Pediatr Surg. 2004;39(9):1376-80.

24. Tarpgaard M, Hansen TM, Rognås L. Anaesthetist-provided prehospital advanced airway management in children: a descriptive study. Scand J Trauma Resusc Emerg Med. 2015;23:61.

25. Prekker ME, Delgado F, Shin J, et al. Pediatric Intubation by paramedics in a large emergency medical services system: process, challenges, and outcomes. Ann Emerg Med. 2016;67(1):20-9.
26. Kerrey BT, Rinderknecht AS, Geis GL, et al. Rapid sequence intubation for pediatric emergency patients: higher frequency of failed attempts and adverse effects found by video review. Ann Emerg Med. 2012;60(3):251-9.

27. Wb T, Salvi N, Orliaguet G, et al. Pro-con debate: cuffed vs non-cuffed endotracheal tube for pediatric anesthesia. Paediatr Anaesth. 2009; 19(Suppl 1):46-54.

28. Bhardwaj N. Pediatric cuffed endotracheal tubes. J Anaesthesiol Clin Pharmacol. 2013;29(1):13-8. 